\title{
注意与非注意位置的知觉组织 ${ }^{*}$
}

\author{
韩世辉 $^{\circledR 囚 * *}$ G. W. Humphreys ${ }^{\circledR} \quad$ 李清阳 ${ }^{(1}$
}

(1) 北京大学心理学系, 北京 100871; (2) 首都师范大学学习与认知实验室, 北京 100037;

(3) Behavioural Brain Sciences, School of Psychology, University of Birmingham, UK)

\begin{abstract}
摘要 研究注意如何影响基于相邻性组织和连接性(UC)的知觉单元的形成. 实验一在视野中 心呈现排成一行的三个由相邻性或 UC 形成的字母, 要求被试识别刺激阵列中位于中间的字母, 刺激与掩蔽之间的 SOA 在 $180 \mathrm{~ms}$ 到 $500 \mathrm{~ms}$ 之间变化. 实验发现在适中的 SOA 条件下, 对相邻 性靶子的反应速度比对 UC 靶子的反应速度慢, 而在长 SOA 和短 SOA 条件下, 对两种靶子的反 应速度没有差别. 与靶子不一致的侧翼字母使得对靶子的反应变慢, 这种侧翼一致性效应在侧翼 刺激是由 UC 决定时比起侧翼刺激由相邻性决定时要大. 实验二研究空间线索提示对相邻性或 UC 靶子的分辨反应时的影响. 与刺激出现在线索提示的位置相比, UC 刺激相对于相邻性刺激的 优势在未被线索提示的位置更明显. 这些结果提示在刺激没有得到完全注意的情况下形成知觉 单元时, UC 具有相对于相邻性组织的优势; 注意对相邻性知觉组织有所助益.
\end{abstract}

\section{关键词 注意 知觉组织 连接性 空间相邻性 相似性}

为了知觉复杂的视觉场景, 人类的知觉系统必 须要把视野中分散的物体组织成组块或知觉单元, 然后对其做进一步的加工. 知觉组织受到相似性、相 邻性和连续性等Gestalt原理的影响 ${ }^{[1]}$. 比如, 空间上 接近的物体容易被组织到一起，相似的物体也容易 被组织到一起. 研究表明, 基于Gestalt原则的知觉组 织对于由空间上分离的局部元素组织成一定的整体 结构是必不可少的 ${ }^{[2,3]}$.

一致连接性(uniform connectedness, UC) 是一个 新的知觉组织原则 ${ }^{[4]}$, 它认为人们倾向于将具有一致
视觉属性(比如亮度、颜色)的连接在一起的区域组织 成一个知觉单元. 实验发现, 与物体是由分离的成分 组成的情况相比，当物体由连通的部分组成时，被试 对两个客体进行匹配的速度 ${ }^{[5]}$ 或者从分心物中辨别 出靶子的速度 ${ }^{[6]}$ 更快. 同样, 被试在同一个连通区域 的两个位置进行知觉判断时的反应时(RTs)要短于在 两个不同连通区域进行时的反应时 ${ }^{[?]}$. 这些结果表明 UC在客体表征和再认中起重要作用.

Palmer等人 ${ }^{[4]}$ 认为UC在Gestalt组织发挥作用之 前形成知觉的最初单元, Gestalt组织的作用则是将这 
些单元分割成低级或者组织成高级的单元, 然后做 进一步的分析. 基于UC的知觉组织要先于基于 Gestalt原则的知觉组织发生. 然而实际的研究结果却 比较复杂. Han等人 ${ }^{[8]}$ 让被试辨别基于相邻性或形状 相似性组织形成的知觉组块的朝向, 被试对相邻性 刺激的反应要快于对相似性组刺激的反应. 这表明 相邻性组织可能早于相似性组织发生. 另外, UC易化 对由相似性或者弱相邻性组织形成的知觉组块的反 应, 这与Pamler和Rock提出的UC优先于Gestalt原则 的假设相一致. 然而, 对基于强相邻性组织形成的刺 激的反应却与基于UC形成的刺激的反应一样快, 即 便在组成相邻性刺激的各单元间的间隔清晰可见的 情况下也仍是如此. Kimchi ${ }^{[9]}$ 也发现知觉组织并不总 是开始于由UC所形成的单元，她让被试将一个启动 刺激与一对测试刺激进行匹配, 这些刺激都是由空 间上分离的局部元素构成的整体形状, 局部元素是 由UC形成的. 当整体形状包含较少局部元素且启动测试间隔较短时, 局部元素的特性决定匹配加工; 而 整体形状由数量较多的元素组成时, 整体结构对匹 配加工影响更大. 这些发现都指出根据整体结构与 组成元素的特性之间关系的不同，用于知觉加工的 初始单元既能由UC形成又能由Gestalt原则(相似性、 相邻性)形成.

我们最近发现UC和相邻性组织决定知觉单元的 效率会受到视野中整体客体的数量的影响 ${ }^{[10]}$. 我们 采用视觉搜索任务, 让被试在分心字母“H”中搜索字 母“E”，这些字母由在空间上分离的局部矩形组成(相 邻性组织)或者是由一致连通的线组成(UC组织). 当 只呈现一两个大字母时, 分心字母与靶子字母组织 原则的不同对反应时(RTs)没有影响. 但是在呈现四 个大字母的情况下, 对由UC形成的靶子的反应要快 于对由相邻性形成的靶子的反应. 我们认为, 在加工 由相邻性决定的刺激与加工由UC决定的刺激时, 注 意所起的作用不同. 当多个项目同时呈现时，用来支 持加工的注意资源相对较少, 而相邻性组织更多依 赖于可供利用的注意资源，因此注意资源的不足对 相邻性组织的影响比对UC组织的影响更大. 如果这 个假设是正确的, 那么我们可以做出如下预期, 与刺 激出现在注意位置时相比, 刺激出现在非注意位置
时UC与相邻性组织间的差别更大, 这就是本研究的 中心问题.

实验一采用Eriksen等人 ${ }^{[11]}$ 的侧翼作业范式研究 在注意和非注意位置上相邻性组织与UC在形成知觉 单元时的差别. 在典型的侧翼作业任务中, 被试在一 行字母中识别出位于中间的那个靶子字母, 而忽略 与靶子同时出现的位于靶子两侧的字母. 当侧翼字 母(若出现在中间)和靶字母被要求做相同反应时, 被 试对靶子的反应较快, 而当侧翼字母(若出现在中间) 和靶字母被要求做不同反应时，被试对靶子的反应 较慢, 这就是侧翼一致效应(flanker compatibility effect, FCE $)^{[12]}$. 一些研究者提出, 侧翼一致效应反映 了对靶子的早期选择注意加工的失败, 对侧翼刺激 也进行了注意加工 ${ }^{[13]}$. 另外一些研究者则认为侧翼 一致效应的大小与对侧翼刺激的注意加工没有关 系 ${ }^{[14]}$, 而是反映了被自动加工的非注意位置的侧翼 刺激特性对靶子加工的影响 ${ }^{[15]}$. 无论是以上哪种情 况, 普遍接受的观点是侧翼刺激并没有得到像对靶 子刺激那样的完全的注意加工 ${ }^{[16,17]}$, 分配到侧翼刺 激上的注意资源要少于分配到靶子上的注意资源. 我们操控形成靶子或者分心字母的知觉组织原则 (UC或相邻性组织), 来判断UC与相邻性效应究竟是 出现在充分注意位置(靶子位置)还是出现在没有得到 充分注意的位置(分心字母位置), 如果这种效应发生 在注意位置, 那么对UC靶子与相邻性靶子整体的反 应时就会不同, 如果这种效应发生在非注意位置, 那 么UC侧翼刺激对分辨靶目标的干扰就会强于相邻性 侧翼刺激.

实验二采用Posner的外周提示线索任务 [18]进一 步检验在注意和非注意位置形成知觉单元时相邻性 组织与UC的相对效应. 在这一范式中, 外周线索可 以出现在靶子将要出现的位置(有效线索), 也可能出 现在其他位置(无效线索). 当线索有效时, 被试对靶

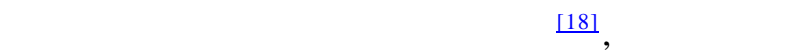
线索引发了刺激驱动的注意. 实验二采取类似的范 式，随机在左视野或右视野呈现一对小线条来指引 注意, 然后三个排成一列的字母出现在注意或非注 意位置, 字母由UC或相邻性组织形成. 实验二比较 在注意位置或者非注意位置呈现靶子时, 被试对 UC 
的靶子和相邻性靶子的反应, 以研究空间注意在对 两种知觉组织加工的影响. 如果减少注意分配会增大 在形成知觉单元时相邻性组织和 UC 组织的差别, 那 么就应该观察到如下情形: 相对于在注意位置时, 对 UC 与相邻性靶子的反应差别在非注意位置时更大.

\section{1 实验一}

实验一采用侧翼作业任务, 考察被试对由相邻 性组织和 UC 所形成的大字母的反应以及 FCE. 实验 一中使用了两组刺激(图 1), A 组刺激图形中的大字 母是由小的长方形组成，而 $\mathrm{B}$ 组刺激中大字母是由实 线组成的, A 组刺激和 B 组刺激的大字母是由不同的 知觉组织原则决定的. 用分辨靶子的反应时反映注 意位置上知觉组织效应的差异, 用 FCE 反映知觉组 织效应在非注意位置的差异. 为了考察 UC 和相邻性 组织之间可能的时间进程上的差异, 我们采用了不 同的刺激到掩蔽之间的 SOA.

\section{1 方法}

(1) 被试: 16 名北京大学的研究生和本科生参与 了实验一, 其中 6 名男性, 10 名女性, 年龄从 18 24 岁, 均为右利手. 被试的裸眼视力或者矫正视力都正 常.

(2) 刺激: 实验一使用了两组刺激, 刺激图形
为黑色, 背景为灰色, 刺激图形组成大写字母 $\mathrm{E}$ 或 $\mathrm{H}$ $\mathrm{A}$ 组刺激的字母由小实心长方形组成, 这些长方形排 列在 $6 \times 7$ 的矩阵中. 在观看距离为 $57 \mathrm{~cm}$ 的条件下, 大字母宽 $2.1^{\circ}$, 高 $2.6^{\circ}$, 每个小长方形宽 $0.25^{\circ}$, 高 $0.28^{\circ}$. 每个相邻的长方形间水平和竖直的距离都为 $0.1^{\circ}$. B组刺激的字母由实线组成, 其中垂直的实心线 条的宽度为 $0.2^{\circ}$, 中间和顶部(及底部)的水平线条的 宽度分别为 $0.12^{\circ}$ 和 $0.15^{\circ}$. B组刺激中的字母大小与 $\mathrm{A}$ 组刺激中的相同. 组成 $\mathrm{A}$ 组刺激字母 $\mathrm{H}$ 和 $\mathrm{E}$ 的小长方 形的面积之和分别为 1.26 和 $1.54 \mathrm{~cm}^{2}$, 组成B组刺激 字母 $\mathrm{H}$ 和 $\mathrm{E}$ 的实心线的面积之和分别为 1.29 和 1.40 $\mathrm{cm}^{2}$. 刺激的排列方式是 3 个大字母排成一行(图 1), 中间的字母由UC或者相邻性组织形成. 侧翼刺激和 靶子是相同的字母时称为一致性条件, 不相同的字 母时称为不一致条件. 两个大字母之间的距离为 $1.8^{\circ}$.

（3）实验程序: 实验采用四因素被试内设计，四 个因素分别为靶子刺激组织原则(排列在刺激中间的 字母由 UC 还是由相邻性组织形成)、侧翼刺激组织 原则(侧翼刺激是由 UC 还是由相邻性组织形成)、一 致性(侧翼刺激和靶子是否是需要相同反应的字母)和 SOA(靶子开始呈现和掩蔽开始呈现的时间间隔, 180, $230,330,500 \mathrm{~ms})$. 实验中每个测试开始有一个作为 注视点的十字呈现在屏幕的中心. 注视点宽 $0.25^{\circ}$,

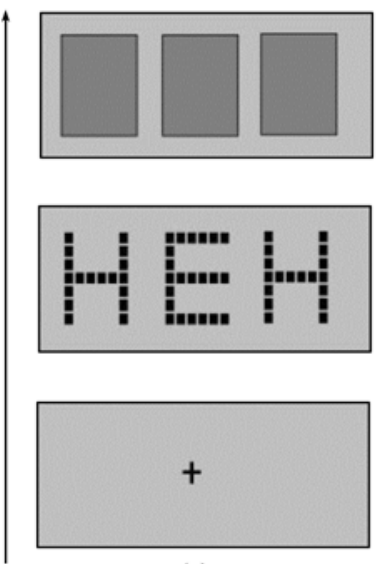

(a)
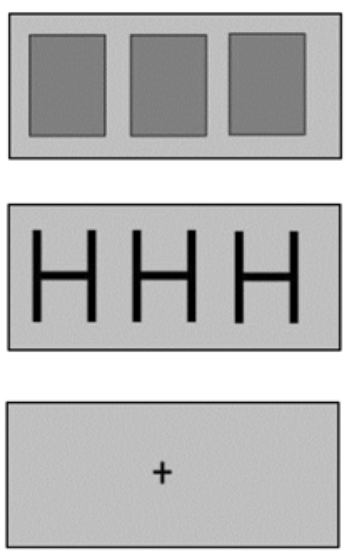

(b)
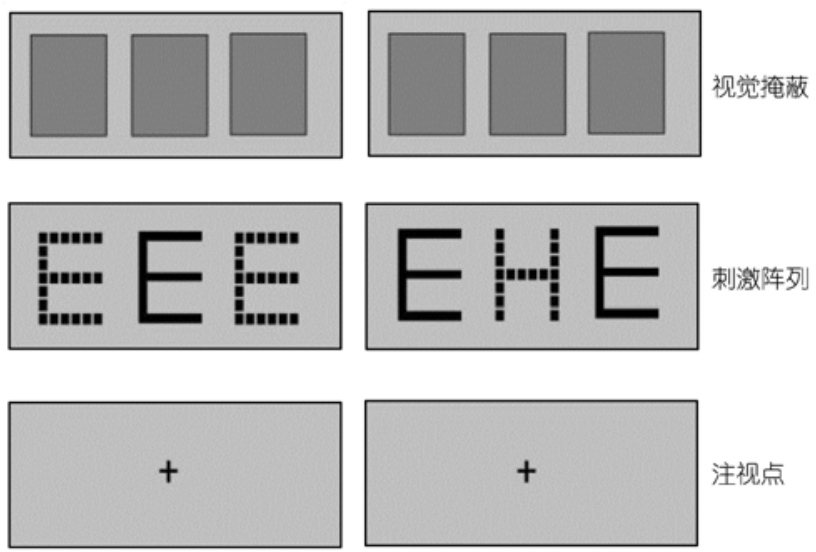

(c)

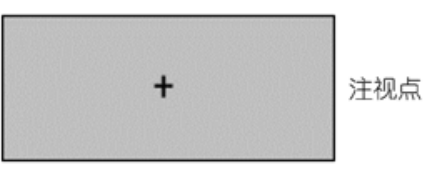

(d)

图 1 实验一中使用的刺激图形

(a)和(b) 靶刺激和侧翼刺激由同一个知觉组织原则定义; (c)和(d) 靶刺激和侧翼刺激由不同的知觉组织原则定义; (b)与(c) 靶刺激和侧翼刺激是一致的; (a)与(d) 靶刺激和侧翼刺激是不一致的 
高 $0.30^{\circ}$, 注视点消失后即在屏幕的中心位置随机呈 现包含有 3 个大字母的刺激图形. 刺激图形呈现时间 为 $150 \mathrm{~ms}$, 然后 3 个灰色的长方形作为掩蔽出现(图 1), 每个长方形宽 $2.4^{\circ}$, 高 $2.9^{\circ}$, 呈现时间为 $200 \mathrm{~ms}$. 刺激和掩蔽之间的 SOA 随机变化在 4 个水平, 180, 230, 330 和 $500 \mathrm{~ms}$. 从一个掩蔽图形消失到下一个刺 激的呈现开始之间的时间间隔(ISI)为 $1300 \mathrm{~ms}$.

在正式实验前, 被试先做 32 次练习. 正式实验 包括 9 组, 每组具有 192 个测试. 一半的测试中, 靶 子字母基于相邻性形成; 另一半测试中, 靶子字母基 于 UC 形成. 每种条件下, 侧翼刺激在一半测试中由 相邻性形成，而另一半测试中则由 UC 形成. 被试的 任务是辨别刺激中央位置的字母 $(\mathrm{H}$ 还是 $\mathrm{E})$, 并通过 按不同的键报告. 标准反应键盘上有两个按键, 分别 代表两个字母. 一半的被试看到 $\mathrm{H}$ 按左键报告, 看到 $\mathrm{E}$ 按右键报告, 另一半的被试则相反. 要求被试尽可 能快而准确地进行反应.
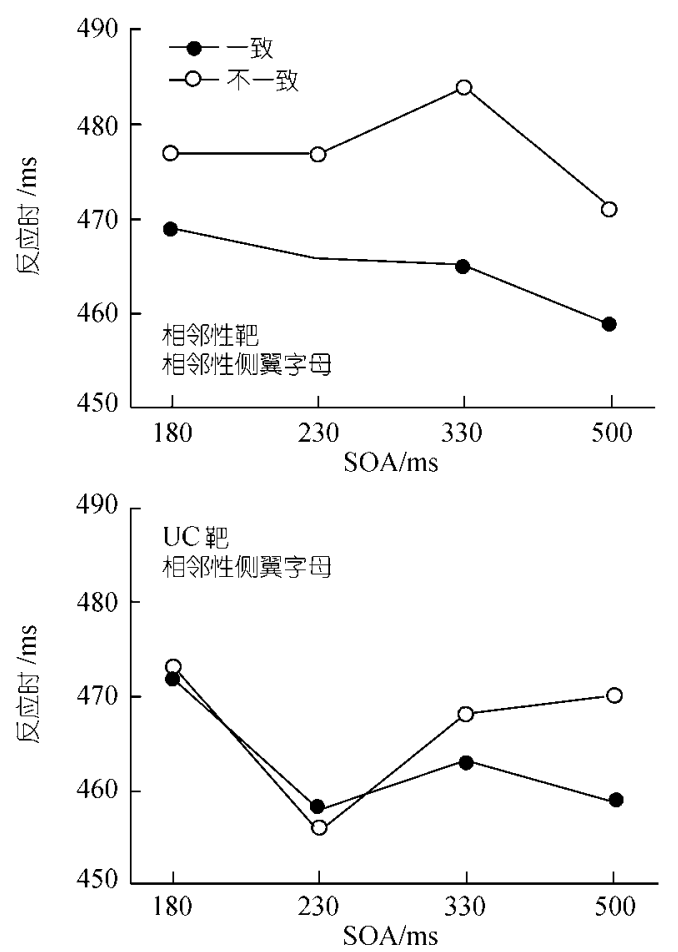

\section{2 结果}

实验一以反应时和错误率作为因变量, 靶子刺 激组织原则、侧翼刺激组织原则、一致性和 SOA 四 个因素为自变量, 进行重复测量的方差分析. 实验一 中各条件下的平均反应时见图 2, 图中上方两个图形 描述了对相邻性定义的靶子的反应时，下方两个图 形则显示 UC 定义的靶子的反应时. 方差分析表明, 4 个自变量的主效应显著, 分别为靶子刺激组织原则 $(F(1,15)=46.22, P<0.001)$. 侧翼刺激组织原则 $(\mathrm{F}(1,15)=13.55, P<0.002)$ 、一致性 $(\mathrm{F}(1,15)=55.90$, $P<0.001)$ 和 SOA $(\mathrm{F}(1,15)=5.94, P<0.002)$. 被试对 相邻性定义的靶子的反应速度要慢于对 UC 定义的靶 子的反应速度. 在侧翼刺激是由相邻性定义的情况 下, 被试对靶子字母的反应要快于由 UC 定义侧翼刺 激的情况, 当侧翼刺激和靶子不一致时被试对靶子 的反应要慢于一致的情况，被试的反应速度也随 SOA 的变化而变化.
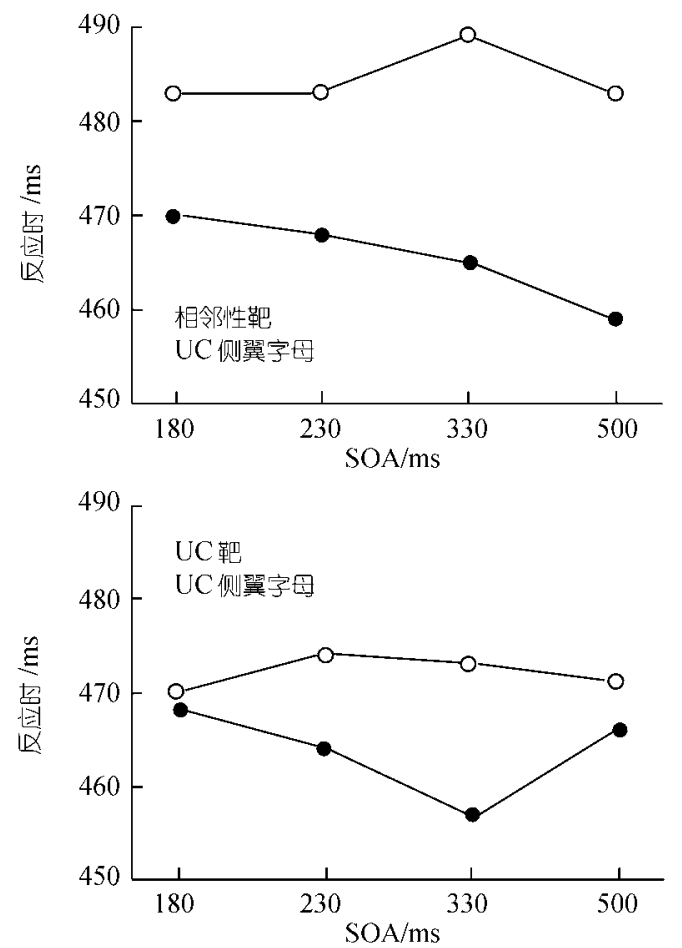

图 2 实验一的反应时结果 上方的图是不同 SOA 情况下对相邻性靼子的反应时结果, 下方的图是对 UC 靶子的反应时的结果 
靶子刺激组织原则和一致性的交互作用显著 $(\mathrm{F}(1,15)=23.22, P<0.001)$, 说明侧翼一致效应在靶 子字母是由相邻性定义时较大, 而在靶子字母是由 UC 定义时较小. 侧翼刺激组织原则和一致性的交互 作用也显著 $(\mathrm{F}(1,15)=4.52, P<0.05)$, 相对于由相邻 性定义的侧翼字母, 由 UC 定义的侧翼字母对靶子的 反应产生了更大的干扰. 靶子刺激组织原则和 SOA 的交互作用也显著 $(\mathrm{F}(3,45)=5.71, P<0.002)$, 在 SOA 很短 $(180 \mathrm{~ms})$ 和很长时 $(500 \mathrm{~ms})$, 对 UC 定义的靶子的 反应和对相邻性定义的靶子的反应之间差别较小; 而当 SOA 处于中等水平时 (230 和 $330 \mathrm{~ms}$ ), 对两种靶 子字母的反应时的差别较大. 比起短 SOA(180 和 230 $\mathrm{ms}$ )的情况, 侧翼刺激在长 SOA(330 和 $500 \mathrm{~ms}$ )情况 下产生了更大的干扰效应，因此一致性和 SOA 的交 互作用也显著 $(\mathrm{F}(3,45)=5.59, P<0.003)$. 事后分析 表明, 在 SOA 中等时(230 和 $330 \mathrm{~ms}$ ), 对 UC 靶子的 反应要比对相邻性靶子的反应快 $(\mathrm{F}(1,15)=29.9, P<$ 0.001 和 $\mathrm{F}(1,15)=236.5, P<0.001)$. 然而, 在短和长 SOA 情况下 (180 和 $500 \mathrm{~ms})$, 不管侧翼刺激由 UC 还 是由相邻性知觉组织形成, 对 UC 靶子的反应时和对 相邻性靶子的反应时没有显著差异 $(\mathrm{F}(1,15)=3.34, P>$ 0.08 和 $F<1$ ). 另外, 当 SOA 为 180,230 和 $500 \mathrm{~ms}$ 时, 对 UC 靶子的反应不受侧翼刺激组织原则这一因 素的影响. 对应的统计量分别为: SOA 为 180 时 $\mathrm{F}<1$, SOA 为 230 时 $F(1,15)=3.34, P>0.08$, SOA 为 500 $\mathrm{ms}$ 时 $\mathrm{F}(1,15)=3.47, P>0.078$. UC 靶子的侧翼一致 效应只在 SOA 为 $330 \mathrm{~ms}$ 的条件下显著, $(F(1,15)=$ $14.9, P<0.002)$. 相反地, 在所有 SOA 条件下, 对相 邻性的靶子的反应都因为不一致的侧翼刺激的出现 而显著地变慢 $(P<0.01)$.

被试反应的错误率较低, 平均错误率为 $3.3 \%$. 以错误率为因变量的方差分析结果表明, 靶子刺激 组织原则主效应显著 $(F(1,15)=5.35, P<0.03)$, 对 UC 靶子反应错误率比对相邻性靶子的反应错误率稍 低(3.0\%和 3.5\%). 一致性的主效应也显著, $(\mathrm{F}(1,15)=$ $7.59, P<0.014)$, 说明在不一致的条件下错误率(3.6\%) 高于一致条件时错误率(3.0\%). 靶子刺激组织原则和 SOA 有显著的交互作用, $(\mathrm{F}(3,45)=9.18, P<0001)$, 比起 SOA 短或长的条件(180 和 $500 \mathrm{~ms}), \mathrm{SOA}$ 中等时
(230 和 $330 \mathrm{~ms}$ ) 对 UC 靶子的反应错误率和对相邻性 靶子的反应错误率之间的差异更大.

\section{3 讨论}

实验一检验在形成大字母时，在注意和不被注 意的位置上相邻性组织和UC相对的有效性. 我们发 现, 对UC靶子的反应显著地快于对相邻性靶子的反 应, 中等SOA的条件下尤其如此. 实验中靶子的出现 总是伴随着分心物, 因此, UC靶子的优势在多项目呈 现时发生, 这与我们之前的研究中发现的UC刺激在 此条件下受益的结果是一致的 ${ }^{[10]}$. SOA的效应表明, 在被注意的位置, UC对相邻性组织的优势随 着时 间变化而显露出来. 在知觉加工的早期(当 SOA短时) 相邻性和UC一样有效, 然而, 随着SOA的加长, UC 在形成知觉单元时逐渐变得更有优势, 直到在 SOA 达到一定长度时, UC的有效性也趋于某一固 定值.

除了靶子间的差异, 侧翼刺激的干扰作用也随 着分心物是否由UC或由相邻性组织形成而不同. 当 分心物由UC形成时, 干扰效应比分心物由相邻性形 成时更大, 同时, 这种干扰效应只同相邻性靶子一起 出现, 这表明侧翼一致效应至少部分地依赖于形成 靶子的刺激编码和形成分心物的刺激编码之间的竞 争关系 ${ }^{[19]}$. UC靶子从这个竞争中胜出, 同时又不容 易收到侧翼刺激的干扰. 当靶子由相邻性定义、分心 物由UC定义时, 分心物对靶子的干扰效应最大. 从 相似性知觉组织的角度来看, 我们得到了另一个不

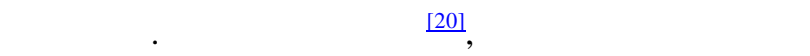
属于不同的知觉组块时相比, 当刺激是同属于某个 整体的一部分时, 侧翼刺激干扰效应较大. 本实验中 在靶子和分心物都是由相邻性定义的或者都是由UC 定义的两种条件下, 都有较强的相似性知觉组织, 但 这两个条件下都没有最大的侧翼一致效应(而且在 $\mathrm{UC}$ 靶子的条件下也没有可靠的侧翼一致效应出现). 本实验中, 最有优势的不是项目 (字母)间的相似性知 觉组织, 而是每个单个项目 (字母)内的知觉组织的速 度. 这一方面可能反映了UC和相邻性知觉组织的有 效性的差别, 它们影响单个项目, 另一方面又反映了 靶子和分心物的相似性组织作用. 此外, UC侧翼刺 激的干扰作用表明，由于对分心物的注意比对靶子 
的注意要少很多, 相邻性知觉组织和 UC 之间的差别 不依赖于对刺激的完全的注意.

\section{2 实验二}

在实验一中尽管没有要求被试去注意侧翼字母, 但由于靶子和侧翼字母相互距离很近, 侧翼一致效 应仍有可能反映了对侧翼刺激字母的部分注意. 实 验二采用线索提示任务将注意位置和非注意位置进 行了区分. 一般认为, 外周线索出现后, 注意加工会 集中在被线索提示的位置. 因此实验二通过在提示 位置和未提示位置上将被试对 UC 和相邻性靶子的反 应进行比较, 来研究空间注意如何影响 UC 和相邻性 组织的差异. 由于实验一的结果显示当靶子和侧翼 刺激被不同知觉组织原则定义时，侧翼刺激干扰作 用有着显著的不同，我们在实验二中只使用了靶子 和侧翼刺激不一致的条件检验在提示位置和未提示 位置上侧翼刺激的干扰作用. 另外，为了使侧翼字母 距离注视点同样远，侧翼字母和靶子被安排在了一 列中 (在实验一中它们是排成行的), 如图 3 所示. 实 验二同时研究了在空间注意和建构知觉单元(基于 UC 和相邻性知觉组织)的交互作用中客体数量的效 应. 为此增加了一个实验条件, 即呈现在提示位置或 未提示位置的只有一个由 UC 或相邻性定义大字母.

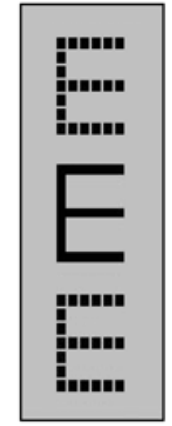

(a)

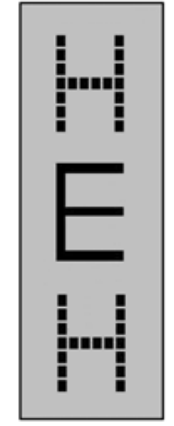

(b) (c)

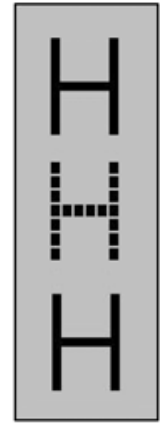

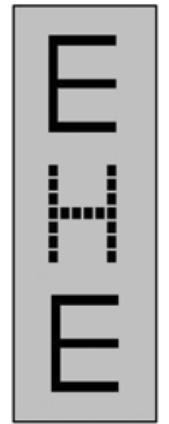

(d)
图 3 实验二中的整体客体条件下的刺激阵列 (a)和(b) 靶刺激由 UC 定义, 侧翼刺激由相邻性组织原则定义; (c)和 (d) 靶刺激由相邻性组织原则定义, 侧翼刺激由 UC 定义; (a)与(c) 靶 刺激和侧翼刺激是一致的; (b)与(d) 靶刺激和侧翼刺激是不一致的

\section{1 方法}

(1) 被试: 16 名北京大学的研究生和本科生参与 了实验二, 其中 8 名男性, 8 名女性, 年龄为 19 28 岁, 均为右利手. 被试的裸眼视力或者矫正视力正常.

(2) 刺激和程序: 实验二大部分与实验一相同, 只有以下所述不同.

在多个整体字母条件下，每个刺激阵列都包括 纵向排成一列 3 个大字母(图 3). 顶部和底部的字母 相同，它们与中间的靶子一致或不一致. 多个字母刺 激组的一半测试中, 刺激图形的顶部和底部的字母 由 UC 定义，同时其中间的靶子由相邻性定义. 另一 半测试中, 安排正相反. 两个相邻大字母的顶部和底 部的边缘间隔为 $2.4^{\circ}$. 多个整体字母条件下的实验采 用四因素被试内设计，四个因素分别为线索有效性 (有效、中性或无效)、靶子字母组织原则(靶子由 UC 或相邻性形成)、一致性(侧翼刺激和靶子是否一致)、 视野(刺激呈现在左或右视野). 单个整体字母条件下, 除了在单个整体字母条件下只呈现中间的那一个大 字母之外，其他所有设计都与多个整体字母条件相 匹配. 单个与多个整体字母条件会出现在相同的实 验组里. 每次测试开始, 屏幕的中心会呈现一个作为 注视点的“十”字. 在有效线索和无效线索的条件下， 两个小横线条呈现在左视野或右视野作为提示线索, 其中一个横线条出现在顶部大字母和中间大字母之 间的一个位置, 另一个线条出现在中间大字母和底 部大字母之间的一个位置上. 每个线条宽 $0.45^{\circ}$, 高 $0.14^{\circ}$. 在中性线索条件下, 提示线索在左右视野同时 出现. 3 种条件下的线索呈现时间都是 $100 \mathrm{~ms}$. 提示 线索消失后 $300 \mathrm{~ms}$, 在左或右视野会出现一个字母 列或单个大字母. 中间大字母的中心位于屏幕的子 午线上, 距离注视点 $5.3^{\circ}$, 刺激呈现时间为 $150 \mathrm{~ms}$. 前一个测试中靶子出现到下一个测试提示线索出现 之间的时间间隔在 1000 1500 ms 之间随机变化.

被试首先做 40 次练习, 然后进行 10 组(每组 160 个测试)正式实验. 实验任务要求被试辨认靶子字母 ( $\mathrm{H}$ 还是 $\mathrm{E})$, 靶子字母在多个整体字母条件下是一个 刺激阵列中间的大字母, 在单个整体客体条件下就 是呈现的那个大字母. $60 \%$ 的测试中, 刺激阵列出现 
在线索提示的位置上(有效线索条件); $20 \%$ 的测试中 刺激阵列出现在和线索提示相对的位置上(无效线索 条件); $20 \%$ 的测试中, 线索在两侧视野中同时出现, 刺激阵列则随机出现在左或右视野(中性线索条件).

\section{2 结果}

实验二以反应时和错误率为因变量. 在多个刺 激的条件下, 线索有效性、靶子字母组织原则、一致 性和视野四个因素为自变量进行方差分析. 在单个 刺激的条件下, 以线索有效性、靶子字母组织原则和 视野三个因素为自变量进行方差分析.

(1) 多个字母条件：对多个字母条件下反应时的 分析结果表明, 线索有效性显著 $(\mathrm{F}(2,30)=33.85, P<$ 0.001), 有效线索条件下的反应时比中性线索条件下 的反应时要更短, 中性线索条件下的反应时则比无 效线索条件下的反应时要短. 靶子字母组织原则也 显示出显著的主效应 $(F(1,15)=70.45, P<0.001)$, 一致 性的主效应也显著 $(\mathrm{F}(1,15)=120.19, P<0.001)$, 这表 明对 UC 靶子的反应快于对相邻性靶子的反应, 不一 致的侧翼刺激减慢了对靶子的反应速度. 线索有效 性和靶子字母组织原则的交互作用显著 $(F(2,30)=$ 3.67, $P<0.036)$, 说明对 UC 靶子和相邻性靶子的反 应之间的差别受空间注意的影响一比起中性线索 条件，在无效线索条件下，二者间差异更大，而中性 线索条件下的二者间差异比有效线索条件下的差异 更大(图 4). 靶子字母组织原则和一致性之间也显示 出显著的交互作用 $(\mathrm{F}(1,15)=21.39, P<0.001)$, 这是因 为不一致的侧翼刺激对相邻性靶子产生了更大的干 扰效应, 而对 UC 靶子的影响较小. 然而, UC 分心物 相对于相邻性分心物的侧翼刺激效应的增加, 却没 有在有效线索、中性线索和无效线索 3 种条件下发 生变化 $(\mathrm{F}<1)$.

多个字母条件下的错误率很低, 平均值为 $4.3 \%$. 方差分析表明, 靶子字母组织原则有显著的主效应 $(\mathrm{F}(1,15)=12.27, P<0.003)$, 这表明被试在对相邻性靶 子反应时比对 UC 靶子反应时犯的错误更多 (5.6\% vs. $3.9 \%)$. 一致性的主效应也同样显著 $(\mathrm{F}(1,15)=15.38, P$ $<0.002)$, 说明比起侧翼刺激和靶子一致的条件, 当 侧翼刺激与靶子不一致时, 被试更容易犯错误.
（2）单个字母条件: 单个字母条件下的反应时和 错误率如图 5 所示. 线索有效性的主效应在反应时作 为因变量时显著 $(\mathrm{F}(2,30)=44.67, P<0.001)$; 错误率 为因变量时也显著 $(\mathrm{F}(2,30)=8.10, P<0.002)$. 相对 于中性线索条件, 有效线索条件下的反应速度更快, 而中性线索条件下的反应比无效线索条件下的反应 要快. 有效线索条件下的错误率比无效线索条件下 的错误率要低, 无效线索条件下的错误率比中性线 索条件下的又低. 然而, 靶子字母组织原则主效应在 以反应时和错误率分别做因变量时都不显著, 线索 有效性和靶子字母组织原则交互作用也不显著 $(P>$ $0.1)$, 说明反应速度和错误率不随 UC 和相邻性靶子 在提示或未提示的位置上而改变.

\section{3 讨论}

实验二首先揭示了靶子加工过程中空间注意的 作用. 当靶子出现在提示位置时, 被试对靶子的反应 比靶子出现在未提示位置时要快, 在单个字母条件 和多个字母条件下的相邻性和UC靶子的反应也存在 着相似的注意效应. 这样的结果与以前的研究结果 一致 ${ }^{[18]}$, 表明不管靶子字母由什么知觉组织原则定 义，空间注意可以促进对靶子的加工.

多个字母条件下的结果证实了被试对 UC 靶子 的反应比对相邻性靶子的反应更快, 这反映出当多 个客体呈现而产生注意竞争时, UC 相对于相邻性在

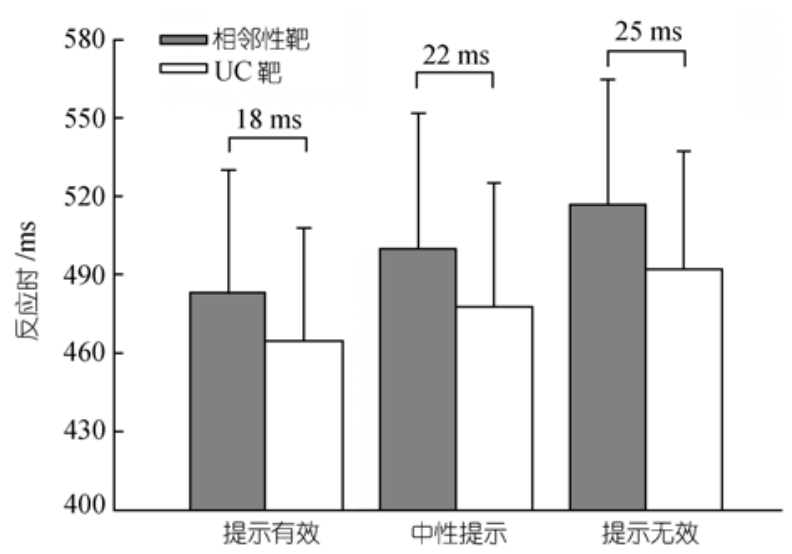

图 4 实验二中多个整体客体条件下的反应时的结果 直方图上方标注的是对相邻性靶子和 UC 靶子的反应速度的差异, 分别对应着有效线索条件、中性线索条件和无效线索条件 

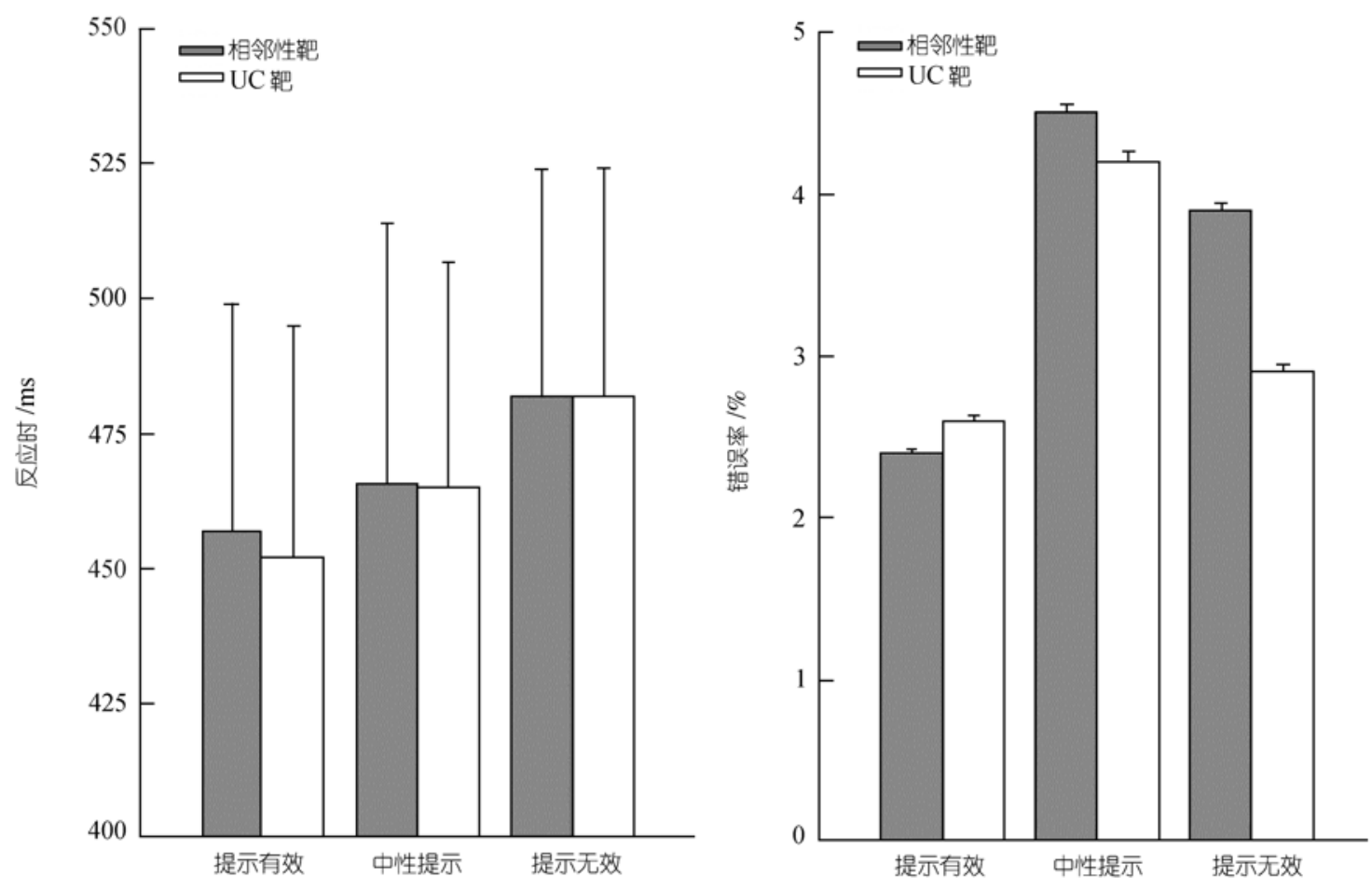

图 5 实验二的单个整体客体条件下反应时和错误率的结果

形成靶子上的优势. 这一点上实验二与实验一的结 果一致, 说明无论刺激是在中央视野呈现还是在外 周视野呈现, UC相对于相邻性组织的优势都存在. 更 重要的是, 实验二的结果证实UC和相邻性在形成靶 子时的差异在刺激出现在非注意的位置上时比刺激 出现在注意的位置时更大. 这些结果支持了如下的 假设: 在多个客体条件下形成知觉单元时, 基于相邻 性知觉组织要比UC需要更多的注意努力 ${ }^{[10]}$. 实验一 中, 不一致的侧翼刺激减慢了对靶子的反应速度, 而 且这种效应在分心物由UC定义时比由相邻性定义时 更大, 我们的数据显示这种干扰效应的增大并不受 空间注意的影响, 这表明在靶子和侧翼刺激位置上, 对相邻性知觉组织的注意效应在与对UC的注意效应 之间的分离. 空间注意调节靶子的形成, 出现在注意 位置的这些靶子被注意选择了. 相对的, 实验没有发 现线索提示和分心物知觉组织的交互作用, 这与分 心物只得到很少的注意 (即使是出现在有线索
提示的位置, 分心物得到的注意也非常少)是相一致 的. 当刺激得到的注意资源很少时, UC知觉组织比相 邻性知觉组织更有优势 ${ }^{[10]}$.

然而在单个客体条件下的注意和非注意的位置 上, 被试对UC和相邻性靶子的反应速度是同样快的, 这一点和我们以前的工作一致 ${ }^{[2]}$. 在我们以前的研究 中, 大字母呈现在注视点的位置, 让被试对由UC或 相邻性组织在相同的空间范围形成的知觉单元进行 识别和再认, 结果并没有发现UC比相邻性知觉组织 更有效率. 单个客体条件与多个客体条件下的结果 的对比提供了更多的证据说明UC和相邻性知觉组织 的差别依赖于注意. 只是当多个整体客体同时呈现、 注意出现竞争的条件下, 形成大字母时UC知觉组织 比相邻性知觉组织更有效的结果才会出现. 当呈现 的是单个整体客体, 没有注意竞争时, 线索 提示对 U C 和相邻性知觉组织的相对有效性的作用 很小. 


\section{3 总讨论}

现在有相当多的证据说明在视觉加工的早期阶 段, 视觉的基本元素被编码形成可能的客体, 然后对 这些客体进行下一步的更高水平上的识别加工 ${ }^{[21]}$. 研究者普遍认为知觉组织发生在注意之前, 因此不 管注意资源在知觉组织发生的空间位置上如何分配, 都不会影响到知觉组织 ${ }^{[22]}$. 如果确实如此的话, 那么 不同知觉组织过程间的差异也不应受到注意分配的 影响, 然而我们的数据并不支持这一推测.

我们的实验结果表明, 相邻性知觉组织和UC在 形成知觉单元时的有效性要受空间注意的影响. 实 验一应用了侧翼作业任务来检验UC和相邻性知觉组 织在形成靶子和侧翼刺激时的相对有效性. 我们发 现对UC靶子的反应速度比对相邻性靶子的反应速度 要快, 同时, UC侧翼刺激比相邻性侧翼刺激对靶子加 工产生的干扰更大，这些结果说明在多个客体同时 呈现的情况下, 无论在靶子位置还是侧翼位置形成 大字母时, UC都比相邻性更具优势. 此外, 我们 还 发现对UC和相邻性靶子的差异在中等SOA时很明显, 但在长或短SOA时不明显, 但是UC对侧翼刺激的干 扰效应并不因 SOA的变化而变化. 靶子的反应速度 受靶子-掩蔽间SOA的影响以及侧翼刺激对靶子的干 扰效应的不同, 说明了UC相对相邻性知觉组织的优 势在非注意位置比在注意位置上更显著. 实验二中, 我们检验了对空间位置的线索提示对被试反应的影 响, 发现当多个客体同时呈现时, UC相对于相邻性知 觉组织的优势在非注意位置上比注意位置上的更加 显著. 实验一和实验二这样的结果并不能简单的通 过感觉水平的因素来解释, 因为我们对UC和相邻性 定义的靶子都做了亮度匹配, 而且, 如果是低水平的 因素决定了对UC和相邻性靶子的行为反应的差异, 那么不管大字母的数目是多少, 最终的结果都应该 是相似的. 而在单个客体呈现的条件下, 对UC和相 邻性靶子的反应并没有明显差别, 说明感觉水平的 因素对本实验的结果贡献很小. 总之, 这些结果支持 下面的假设，即在多个客体呈现的情况下，用相邻性 知觉组织去构建知觉单元比用UC构建时需要更多的 注意努力 ${ }^{[10]}$.
如果知觉组织独立于空间注意的话, 我们的这 些结果就难于解释. 那么, 考虑其他一些研究结果说 明知觉组织可以在刺激未被注意到的时候就可以发 生 ${ }^{[22]}$, 我们应该如何解释本研究的结果? 一种可能 的解释是, 知觉组织可以在没有注意的参与时就可 以启动, 但是它又可以被随后的注意的反馈所影响, 这与神经生理学的发现一致. 有证据表明, 在初级视 觉皮层注意对知觉组织有晚期激活效应 ${ }^{[23]}$. 这种解 释与行为数据一一知觉组织在当被试被第二种任务 分散注意力时减弱——相一致 ${ }^{[24]}$. 我们的结果表明, 基于相邻性知觉组织和UC的知觉单元的形成过程对 注意资源的要求是不同的: 比起相邻性知觉组织, UC 要求的注意资源更少. 脑功能成像的研究结果进一 步显示, 比起由其他知觉组织因素(例如共线性)决定 的刺激, 被UC定义的刺激在视觉皮层区域产生的激 活更强 25$]$. 根据这样的结果可以推断, 基于相邻性知 觉组织和UC的知觉单元的形成对注意资源的不同需 求可能反映了在早期视觉皮层用UC对靶子进行定义 是不受注意影响的, 但是这个推断还需要更多实验 的证实.

本研究的数据也与知觉组织的层次理论相一致, 这种理论假定某些形式的知觉组织可能比其他形式 的知觉组织完成得更早. 以前的行为研究表明, 对相 邻刺激的反应比对相似性刺激的反应要快 ${ }^{[2,3,8]}$, 最近 有关相邻性和相似性知觉组织的事件相关电位研究 也支持这个结论 ${ }^{[26]}$. 这些研究发现, 相邻性知觉组织 首先被刺激呈现后 100 到 $120 \mathrm{~ms}$ 之间的一个位于中 央枕叶皮层的正的电活动所标示, 随后在刺激呈现 后 $180 \mathrm{~ms}$ 时会出现枕-顶负波, 这一负波在右半球幅 度更大. 伴随相似性知觉组织的是刺激呈现后 260 $m s$ 出现的一个长潜伏期的枕-颢负波, 且在左半球幅 度更大. 这些发现都说明, Gestalt原则定义的不同知 觉组织过程可能具有不同的神经基础和时间进程.

总之, 本研究的结果表明, 当单个客体呈现时 UC 和相邻性知觉组织对形成靶字母的贡献没有区别. 然而当多个客体同时呈现时，不管刺激排列呈现在 视网膜中央凹附近还是外周视野，也不管其呈现在 注意位置和非注意位置上, UC 都比相邻性知觉组织 更有优势, 并且, UC比相邻性知觉组织的在非注意 
位置条件下的优势导致了 UC 定义的侧翼刺激的干扰 效应更强, 这种增强在各种 SOA 情况下(实验一)和当 注意被提示到靶子上时(实验二)都存在. 相反, 在单 个客体呈现即注意资源没有竞争分配时, 相邻性知 觉组织和 UC 在定义靶子时具有相同的有效性. 视觉 空间注意可以调控知觉组织, 但是调控作用随提示 线索的呈现与否而变化.

\section{致谢 感谢陈峰和余一超进行的数据收集工作.}

\section{参 考 文 献}

1 Wertheimer M. Untersuchungen zur Lehre von der Gestalt: II . Psychologische Forshung, 1923, 4: 301 350

2 Han S, Humphreys G W, Chen L. Uniform connectedness and classical Gestalt principles of perceptual grouping. Perception \& Psychophysics. 1999, 61: 661 674

3 Han S, Humphreys G W. Interactions between perceptual organization based on Gestalt laws and those based on hierarchical processing. Perception \& Psychophysics, 1999, 61: 1287 1298

4 Palmer S, Rock I. Rethinking perceptual organization: The role of uniform connectedness. Psychonomic Bulletin \& Review, 1994, 1: 29 55

5 Van Lier R, Wagemans J. Effects of physical connectivity on the representational unity of multi-part configurations. Cognition, 1998, 69: 1 9[DOI]

6 Saiki J, Hummel J E. Connectedness and the integration of parts with relations in shape perception. Journal of Experimental Psychology: Human Perception and Performance, 1998, 24: 227 251[DOI]

7 Watson S E, Kramer A F. Object-based visual selective attention and perceptual organization. Perception \& Psychophysics, 1999, 61: $31 \sim 49$

8 Han S, Humphreys G W, Chen L. Parallel and competitive processes in hierarchical analysis: Perceptual grouping and encoding of closure. Journal of Experimental Psychology: Human Perception and Performance, 1999, 25: 1411 1432[DOI]

9 Kimchi R. The perceptual organization of visual objects: a microgenetic analysis. Vision Research, 2000, 40: 1333 1347[DOI]

10 韩世辉, Humphreys G W. 连接性和空间相邻性在知觉组织的 关系. 中国科学, C辑, 2002, 32(5): 471 480[摘要] [PDF]

11 Eriksen B A, Eriksen C W. Effects of noise letters upon the identification of a target letter in a nonsearch task. Perception \& Psychophsics, 1974, 16: 143 149
12 Miller J. Priming is not necessary for selective-attention failures: Semantice effects of unattended, unprimed letters. Perception \& Psychophysics, 1987, 41: 419 434

13 Yantis S, Johnston J C. On the locus of visual selection: Evidence from focused attention tasks. Journal of Experimental Psychology: Human Perception and Performance, 1990, 16: 135 149[DOI]

14 Schmid P A, Dark V J. Attentional processing of "unattended" flankers: Evidence for a failure of selective attention. Perception \& Psychophysics, 1998, 60: 227 238

15 Miller J. Priming is not necessary for selective-attention failures: Semantice effects of unattended, unprimed letters. Perception \& Psychophysics, 1987, 41: 419 434

16 Johnston W A, Dark V J. In defense of intraperceptual theories of attention. Journal of Experimental Psychology: Human Perception and Performance, 1982, 8: 407 421[DOI]

17 Kahneman D, Treisman A. Changing views of attention and automaticity. In: Parasuraman R, Davies D R, eds. Varieties of Attention. New York: Academic Press, 1984. 29 61

18 Posner M I. Orienting of attention. Quarterly Journal of Experimental Psychology, 1980, 32: 325

19 Eriksen C W. The flanker task and response competition: A useful tool for investigating a variety of cognitive problems. Visual Cognition, 1995, 2: 101 118

20 Baylis G C, Driver J. Visual parsing and response competition: the effect of grouping factors. Perception \& Psychophysics, 1992, 51: $145 \sim 162$

21 Duncan J, Humphreys G W. Visual search and stimulus similarity. Psychological Review, 1989, 96: 433 458[DOI]

22 Moore C M, Egeth H. Perception without attention: Evidence of grouping under conditions of inattention. Journal of Experimental Psychology: Human Perception and Performance, 1997, 23: 339 352[DOI]

23 Gilbert M, Ito M, Kapadia M, Westheimer G. Interactions between attention, context and learning in primary visual cortex. Vision Research, 2000, 40: 1217 1226[DOI]

24 Ben-Av M B, Sagi D, Braun J. Visual attention and perceptual grouping. Perception \& Psychophyscis, 1992, 52: 277 294

25 Gerlach C, Aaside C T, Humphreys G W, Gade A, Paulson O, Law I. Brain activity related to integrative processes in visual object recognition: Bottom-up integration and the modulatory influence of stored knowledge. Neuropsychologia, 2002, 40: 1254 1267[DOI] Han S, Song Y, Ding Y, Yund E Y, Woods D L. Neural substrates for visual perceptual grouping in human. Psychophysiology, 2001, 38: 926 935 